\title{
The Context Completion and Clarification of English Translation of Chinese Classics
}

\author{
Fang Liu \\ Xi'an University of Arts and Science, Xi'an, Shaanxi, 710076
}

Keywords: Chinese classics; English translation; context filling; clarity

\begin{abstract}
Chinese and English language systems can be divided into different levels of cultural context. Chinese language has obvious recessive features, and its flexible features in grammatical structure are more obvious, while English language can achieve more accurate information. The conveyance of the syntactic structure is relatively obvious. In the process of translating Chinese classics into English, it is essential to analyze the context of speech itself, and pay attention to the information asymmetry and information gaps in it, so as to ensure that we can speak in the process of actually carrying out translation activities. The environment is effectively supplemented, and the translation effect is improved by using clear means to ensure that the translation can meet the reading needs of the audience.
\end{abstract}

\section{Introduction}

From the perspective of high and low context culture, the Chinese and English languages are analyzed. It is found that the low level context is mainly that the language itself can realize the clear communication of a large amount of information. In the high level context, most of the information exists in the cultural exchange. In terms of information, the transmission of information has a hidden character. Therefore, in the process of translating Chinese classics belonging to high-context culture, it is necessary to comprehensively examine the situation of filling in the context, and pay attention to the transfer rationality and logicality of information to ensure the actual effect of the translation work. The translated literature can be highly recognized and affirmed by the target audience, and effectively promote the better dissemination of culture.

\section{The Necessity of Gap Filling in the English Translation of Chinese Classics}

The language of Chinese classics is rather embarrassing, and the concealing characteristics of information are obvious. Especially for the literary classics and philosophical classics in Chinese classics, the high-context cultural characteristics are more obvious, generally for the ancient Chinese readers, and the existence a large amount of information can be reflected in the language of the time. However, after the great changes in time and space, these languages are not only relatively different in terms of grammar and modern Chinese. Modern Chinese scholars have certain difficulties in understanding. For foreign readers of ancient culture, understanding is more difficult, and even foreign scholars have no way to start research. In the process of translating Chinese classics, in order to restore the main ideas of the text, it is necessary to separate the original text from the corresponding context and place it in a new context. Therefore, only comprehensive investigation the context of the original text and the context of the target language can enhance the translation effect and avoid the situation of high and low contextual culture. From this perspective, when translating Chinese classics into English, it is necessary to analyze the connection between Chinese and English and context, and explore the measures to fill the gap on the basis of comprehensive investigation. Make sure you can actually enhance your translation. In other words, there are a large number of sentences, syntaxes and other information in the Chinese system that cannot be revealed in explicit vocabulary or sentences, but hidden in deep context, while English syntactic features are more logical and highly valued. Prominent form and function, a large amount of information is properly distributed at the linguistic level. Therefore, in the process of developing 
Chinese-English translation, it is necessary to attach great importance to possible information asymmetry and context vacancy, in the comprehensive investigation of vacancies and social cultural vacancies. On the basis of contextual vacancy and loose information vacancies, the use of contextual vacancies is used to adjust information, fill in information and make up for the occurrence of contextual vacancies, thereby effectively improving the practical effect of translation work of Chinese classics and ensuring further improvement of translation work. The overall quality.

\section{Translational Clarification Strategy in the English Translation of Chinese Classics}

The clarification strategy in translation is mainly based on a comprehensive interpretation of the original information, and the main information is presented in a relatively clear way in the translation process. The clarification of the English translation of Chinese classics mentioned in this research work not only involves the use of explicit means in the form of language articulation, but also the clarification of the meaning level, that is, the translation of the sentences in the translation The meaning can be more accurately conveyed and enhance the logic and specificity of the translation language.

In the process of carrying out translation activities in specific organizations, translators must systematically analyze the differences in Chinese thoughts, behaviors, and expressions in order to form a deeper understanding of language differences. In the practical exploration activities of implementing English translation for Chinese classics, it is necessary to use a variety of different translation methods in combination with different texts, so as to improve the translation effect while taking into account the differences between the two cultures, and truly realize the contextual filling and translation clarity. Goal. The following is a detailed analysis of the means of translation clarification, combined with the addition, transformation, concrete and other means to systematically analyze the specific case as the entry point, and effectively improve the value of translated text, and then assist China's traditional culture in the world. Effective dissemination within.

Addition is an appropriate addition in the process of implementing English translation of Chinese classics. It is difficult to understand for the original readers but it is difficult for the target readers to understand. It ensures effective translation of translation activities under the guidance of relevance translation theory. . In order to obtain the best correlation in the supplementary translation activities, the translator should pay attention to the system analysis to supplement the information, create the corresponding context for the target language readers to read the relevant text, and ensure that they can deepen the meaning of the language in a specific context. Learn and understand, and then grasp the logic of the text and the connotation of the text from a deeper level. In the process of using supplementary means for translation, the main methods include supplementary connectives, supplementary sentence ellipsis, and supplementary sentence background information, and there are obvious differences in the effects achieved by different supplemental methods [1].

Taking the supplementary sentence ellipsis as an example, in the process of translating Chinese classics, it is necessary to clearly recognize that the frequency of sentence component ellipsis in Chinese classics is high, and because Chinese culture has a high context, it can be omitted in sentences. Subjects, even objects, predicates, etc. can be omitted. In English sentences, subject and predicate verbs are indispensable components. Therefore, when translating Chinese classics, translators must first clarify the omitted elements in Chinese, and then appropriately supplement them in English translations. The actual effect of Chinese translation of English classics. For example, in "Cai Gen Tan", "A man with only slight experience of the world will likewise", in the translation of Paul White, "the world is shallow, the dyeing is shallow; the history is deep, the machinery is deep." Be only slightly stained with its impurities......." Use "A man" as the subject to supplement, so that the sentence composition is complete, and the meaning of the original text can be fully expressed.

The translation translation method is a method to effectively deal with the pragmatic problems in translation. It is mainly used in the process of translating the names, place names and social and cultural customs in the original texts. Meet the requirements of cultural differences to comply with 
the theory. In the practice exploration activities of English translation of Chinese classics by means of conversion, the choice of language becomes a possibility, and the negotiation translation strategy greatly enhances the flexibility of language use and can also promote the realization of ideal communication realm [2]. On the basis of using translation means for translation processing, it can also eliminate the strangeness and strangeness of the target language readers in reading the translated text and understanding the culture of other countries, and effectively assist the in-depth advancement of cultural communication work.

For example, in the "The Analects of Confucius - The Sixth Chapter of the Analects of Confucius", "Zi Zi: "There is no wish to marry, but the beauty of the Song Dynasty, ...". When translating, the translation of the special term "Zhu" is needed. The conversion method is used for proper translation. For example, in the translated text of Gu Hongming, it will be converted into the famous British writer Sydney in the 19th century, so that the reader can grasp the relationship between the text contents in the process of reading, and thus form a deeper content of the original. Level of understanding [3].

In the process of translating the meanings of the meanings into English, it is necessary to ensure that the selected texts in the translations are not affected and bound by the words in the original text, and the meanings in the original texts are directly interpreted to ensure that they are in France. Under the guidance of the interpretation theory, it is possible to better explain the ideological connotation in the original text and better promote cultural exchanges. At this stage, in the process of translating some idioms, allusions, metaphors, etc. in Chinese Chinese classics, the method of interpretation has been used, and a good translation effect has been achieved [4].

For example, in the process of translating "Treaty of the Great Powers, If Cooking", in the "Traditional Chapters of Morals", translators will recognize the ambiguity of the original language, which may result if not explained in detail. It is difficult for the target language reader to understand the thoughts in the reading process. Therefore, the text can be directly interpreted in the translation process. For example, in Arthur Waley's translation, the commentary is "the less one han- dles them the better”, which not only facilitates the reader's reading and understanding, but also allows the target readers to form a clearer system for Laozi's “doing nothing”. The understanding and comprehensive enhancement of the scientific and rational work of Chinese classics [5].

The specific means of translation is to translate the relatively general vocabulary in the original text and the vocabulary and phrases with high degree of abstraction in the specific translation activities, and also to have the symbolic characteristics in the original text. Make effective conversions to ensure a close connection between the translated text and the original text, and improve the readability of the translated text [6]. The main basis for the use of specific translation methods is the examination of the differences in rhetoric and expression habits between the two languages.

Taking Mr. Wang Hong's translation of "Mozi" as an example, he pointed out that "the husband who is good at attacking and slashing, he said: "I am not in the process of gold jade, children, and land, but..." Mr. Wang Hong fully realized that the meaning of the word "children" in the original text of Mozi is vague. In order to ensure that the translated text can be read and understood by foreign readers, it is recognized by foreign readers and used in the translation process. The specific and clear means are dealt with and translated into concrete courtiers and waiting women, which effectively avoids the translation readers' equivalent of sons and daughters. The English translation of Chinese classics is further enhanced, which can effectively assist Chinese culture in the world. Propagation within [6].

Domestication is a more important means in translation activities. It mainly uses the language that has similar meaning to the original words but has obvious differences in cultural colors to achieve the substitution of the original words and achieve the natural equivalence in the translation process. To ensure that the translation can fully display the meaning and style of the original text. In the process of translating Chinese classics into English, the main purpose of using the domestication translation method is to enable the target language reader to produce a response similar to that of the original reader reading the original text in the process of reading the translation [7]. For example, 
in the process of translating "Cai Gen Tan", combined with White's translation analysis, in the translation of "the heart is not dyed, the desire is the fairy capital", because "Xian" belongs to Taoist language, special reference In the social context of the time, he continued to cultivate to obtain undead people, and also refers to some important people who can reach a special realm. The "Xiandu" is a place where immortals live. In White's translation, "as long as the heart is not fame." And the desire of wealth is polluted, then even if a person lives in a secular world full of basic desires, it will be like living in paradise." [8] The main meaning is presented, using "...it will be like living in Paradise "Paradise refers to Xiandu, explaining the religious language in Chinese classics in Western religious language, not only the understanding of the target language readers, but also the readers' formation of ideological resonance in the reading process and the improvement of the science of translation work." Sex and effectiveness.

\section{Conclusion}

In summary, the English translation of Chinese classics is an inevitable choice to promote the worldwide spread of Chinese traditional culture. Therefore, it is necessary to systematically study and analyze the English translation of Chinese classics, and grasp the huge differences in Chinese and English language context. Appropriate adjustments to the translation strategy ensure that the quality of English translation of Chinese classics can be effectively improved in the process of actually carrying out translation activities, so that foreigners can better understand the conditions for creating traditional Chinese culture and effectively improve the effect of Chinese cultural communication.

\section{References}

[1] HAN Mengqi. The Context Completion and Clarification of English Translation of Chinese Classics [J]. Shanghai Translation, 2016(4):73-76.

[2] Bai Shuchen. Analysis of Contextualized Realization in English Translation of Chinese Classics__ Taking English Translation of Characters in Strange Tales from a Lonely Studio as an Example [J]. Global Human Geography, 2014(18).

[3] Huang Haixiang. Contextual Reconstruction and Cultural Misunderstanding of English Translation of Classics in High and Low Cultural Context-Taking the Contextual Reconstruction of Mair's English Version of Sun Tzu's Art of War and Nine Lands as an Example[J]. Journal of Agricultural University (Social Science Edition), 2014, 12(2): 59-69.

[4] Zhou Jie. From the "Tao Jing" to the translation of the ecological ethics awareness of the translators of the classic translations [J]. Journal of Chengdu Aviation Vocational and Technical College, 2016, 32(2): 14-17.

[5] Cui Ying. The Predatory Analysis of the Textual Genre and Cultural Context of English Translation of National Classics [J]. Journal of Dalian Nationalities University, 2013, 15(4): 391-393.

[6] Xing Nan. The Difficulties and Countermeasures of English Translation of Minority Classics under the Influence of Cultural Context [J]. Guizhou Ethnic Research, 2017(10): 157-160.

[7] $\mathrm{Hu}$ Zongfeng, Ai Fuqi. English Translation of Exegesis and Classics-Taking English Translation of the Analects as an Example [J]. English Research, 2016(1): 76-82.

[8] Wang Hong, Mei Yangchun. A Study of English Translation of Chinese Classics in the Western World__A Review of English Translation of Chinese Classics Based on Reading Expectations of Western Readers [J]. Foreign Languages Research, 2017(1):78-84. 